\title{
Avaliação formativa das competências e habilidades: instrumentação para jogos digitais
}

\author{
Glauber Galvão de Araujo, UFRN - glauber.galvao@gmail.com \\ Eduardo Henrique da Silva Aranha, UFRN - eduardoaranha@dimap.ufrn.br
}

\begin{abstract}
Resumo. O processo formativo necessita de avaliações contínuas e sistemáticas que reflitam o desenvolvimento individual do aluno durante o processo ensino-aprendizagem. A prática da avaliação formativa da aprendizagem tem dinâmicas que permitem observar o aluno e identificar deficiências no seu aprendizado durante o processo de ensino, ainda em tempo de executar ações de ajustes. Nesse sentido, este artigo apresenta, em forma de proposta conceitual, um processo sistêmico de instrumentação da avaliação formativa que explora competências e habilidades almejadas em planos de ensino, para implementação em ambientes computacionais de jogos digitais, com a finalidade de estimular o uso mais frequente dessa função da avaliação no cotidiano dos ambientes de aprendizagem, sejam eles presenciais ou virtuais.
\end{abstract}

Palavras-chave: avaliação formativa, jogos digitais, aprendizagem, método de avaliação.

\section{Formative assessment of the competencies and skills: instrumentation for games}

\begin{abstract}
The formative process requires continuous and systematic assessments that reflect the individual development of the student during the teaching-learning process. Formative assessment of learning has in its practical application dynamics that allow you to observe the student and identify deficiencies in their learning during the teaching process, still in time to perform actions of adjustments. In this direction, this paper presents, in the form of conceptual proposal, a systemic process instrumentation formative assessment that explores competencies and skills in teaching plans, for implementation in computer games environments; with the purpose of stimulate more frequent use of this function assessment in everyday learning environments, whatever they are.
\end{abstract}

Keywords: formative assessment, games, learning, assessment method.

\section{INTRODUÇÃO}

A construção do conhecimento do aprendiz precisa ser regulada a partir de uma observação da sua formação individualizada ao longo do processo ensino-aprendizagem. O professor deve ter empatia e uma imagem adequada do que se passa na mente do seu aprendiz. Sem esse exercício, "há pouca chance de sua intervenção ser decisiva na regulação da aprendizagem" (Perrenoud, 1999). Por regulação da aprendizagem entendese o modo de garantir que o aprendiz receba o apoio do professor para sanar possíveis deficiências de aprendizagem ainda durante o processo de ensino-aprendizagem mediante informações coletadas das avaliações formativas.

Dados coletados por meio de avaliações contínuas, como as que têm função formativa, se concentram principalmente na melhoria do processo ensino-aprendizagem 
e são capazes de refletir a formação do aluno individualmente, subsidiando o professor com informações para sua regulação (Jorba e Sanmarti, 2003).

Contudo, avaliar a formação do aluno durante todo o processo ensinoaprendizagem não é uma tarefa fácil, ainda mais avaliar individualmente em meio a turmas grandes e heterogêneas. Diante dessa dificuldade, a grande maioria dos professores preferem as avaliações que classificam os alunos por conceito depois do processo de ensino (Hadji, 2001), ou seja, avaliações instrumentadas por provas que medem o conhecimento de conteúdo.

Os professores argumentam que faltam recursos pedagógicos suficientemente diversificados que os auxiliem na geração de dados e tempo para interpretá-los, como também a realização das intervenções regulatórias no momento apropriado (Perrenoud, 1999; Quinquer, 2003). Para serem críveis, as avaliações formativas necessitam de instrumentos capazes de coletar dados de formação individualizados, de fácil operacionalização para os professores, em tempo de haver regulação da aprendizagem.

Essa problemática motivou o desenvolvimento de um processo sistêmico, no domínio da avaliação da aprendizagem, para apoiar a avaliação formativa de competências e habilidades com sua instrumentação no ambiente computacional de jogos digitais. Os jogos digitais divertem, desafiam, criam mundos onde os jogadores imergem e vivem aventuras e vários tipos de emoções. Têm componentes computacionais que, orientados por modelos pedagógicos, podem coletar dados valiosos sobre as habilidades, competências, atitudes, reações e o conhecimento dos jogadores e ainda diverti-los.

Nesse contexto, espera-se que a sistematização desse processo sistêmico contribua, no âmbito pedagógico, com os professores na atividade de avaliar continuamente, e com os alunos na obtenção de resultados mais coerentes com os seus desempenhos. Além disso, espera-se ainda que o jogo digital proporcione um ambiente de avaliação mais lúdico, e desse modo, menos cansativo para quem aplica e para quem é avaliado. Com o objetivo de validar as contribuições mencionadas, está em fase de desenvolvimento um jogo digital para aplicação em instituições de ensino básico e superior.

Este artigo dá continuidade a outro publicado no WAvalia 2013, no qual se detalhou o componente "Instrumentar" do framework proposto, considerando a sua relação com a taxonomia de Bloom revisada aplicada a um exemplo prático.

\section{AVALIAÇÃO FORMATIVA}

A avaliação da aprendizagem tem duas funções básicas: (a) uma de seleção e classificação, que ocorre depois do ensino, conhecida como somativa; (b) outra de ajuste do processo de ensino-aprendizagem, de reconhecimento das mudanças, representadas por duas avaliações conhecidas por diagnóstica e formativa e que ocorrem antes e durante o ensino, respectivamente (Jorba e Sanmarti, 2003).

A avaliação formativa, que ocorre continuamente durante o processo de ensino, tem como resultado informações que orientam a ação do professor no sentido de adaptar sua prática e os conteúdos às necessidades dos aprendizes. Quinquer (2003) afirma que, a avaliação formativa atua como "o instrumento adequado para regular e adaptar a programação às necessidades e dificuldades dos estudantes".

Para Perrenoud (1999), a avaliação formativa "introduz uma ruptura, porque propõe deslocar a regulação ao nível das aprendizagens e individualizá-la”. É uma avaliação que se interessa mais pelo processo do que pelos resultados e persegue, segundo Jaume Borna (2003), "a regulação pedagógica, a gestão dos erros e a consolidação dos êxitos". 
A avaliação formativa da aprendizagem gera dados úteis para a adequação das atividades de ensino-aprendizagem às necessidades dos aprendizes (Jorba; Sanmarti, 2003) e, dessa maneira, melhora a qualidade do ensino, aproximando-se mais das necessidades de cada aluno, considerando a sua singularidade, procurando superar a perspectiva de um modelo de ensino "tamanho único" que é demais para alguns e insuficiente para outros.

\section{COMPETÊNCIAS E HABILIDADES}

Para Perrenoud e Thurler (2002, apud Behar e Silva, 2012) a competência é o emprego da consciência de modo rápido e criativo para enfrentar situações que surgem. Segundo Zabala e Arnau (2010, apud Behar e Silva, 2012), para fazer frente a essas situações, é necessário a mobilização de atitudes, habilidades e conhecimentos concomitantemente em uma ação conjunta e inter-relacionada, concepção adotada para a abordagem que envolve jogos digitais como ambiente de instrumentação de avaliações formativas.

A competência é a faculdade de mobilização de um conjunto de recursos cognitivos como saberes, habilidades e informações para solucionar com pertinência e eficácia uma série de situações (Perrenoud, 1999).

As habilidades são elementos pelos quais se pretende atingir as competências. Os alunos demonstram o que sabem e podem aprender através das habilidades, adquiridas por meio dos conteúdos estudados. A prática contínua de habilidades é capaz de expressar competências para a solução de desafios nos domínios da linguagem, da matemática e dos fenômenos naturais, bem como o enfrentamento de situações-problema e a construção de argumentações consistentes. Assim, as habilidades são tanto as que apresentam processos mentais/cognitivos como motores e técnicos (Perrenoud, 1999).

\section{TAXONOMIA DOS OBJETIVOS EDUCACIONAIS}

A Taxonomia dos Objetivos Educacionais, ou ainda, Taxonomia de Bloom, foi criada como uma tentativa de classificar as expectativas quanto à aquisição de conhecimento dos alunos. A classificação foi concebida como um meio de padronizar os objetivos educacionais entre o corpo docente em várias universidades. O principal nome nessa tarefa foi de Benjamin S. Bloom, Diretor adjunto do conselho de exames da Universidade de Chicago, que iniciou a ideia, esperando conseguir reduzir o trabalho na preparação dos alunos para os "anual comprehensive examinations". Sua versão final foi publicada em 1956 sob o título original "Taxonomy of Educational Objectives: The Classification of Educational Goals. Handbook I: Cognitive Domain" (Bloom, Engelhart, Furst, Hill, \& Krathwohl, 1956). As ideias de Bloom sobre o domínio cognitivo, expressas em seis níveis do raciocínio, foi amplamente adotada e usada em inúmeros contextos (Krathwohl, 2002).

Segundo Pimentel (2006), Bloom considerou que uma taxonomia poderia facilitar a troca de informações no contexto do desenvolvimento curricular e dos planos de avaliação. Desse modo, os processos cognitivos foram organizados em uma lista do mais simples ou concreto, que é ter a informação, ao mais complexo ou abstrato, que implica julgamento sobre o valor e a importância de uma ideia: (1) conhecimento; (2) compreensão; (3) aplicação; (4) análise; (5) síntese e; (6) avaliação.

Um dos críticos da taxonomia de Bloom, Lorin Anderson et al. (2001, apud Pimentel, 2006), aluno de Bloom, argumenta que praticamente todas as atividades de ensino complexas requerem o uso de várias capacitações cognitivas diferentes, o que para 
a taxonomia original é vista como uma classificação unidimensional. Desse modo, Anderson propôs duas dimensões nos objetivos educacionais: uma dimensão para o conhecimento e outra para os processos cognitivos. Desse modo, a taxonomia de Bloom revisada, como ficou conhecida, tentou corrigir alguns problemas da taxonomia original e diferencia "saber o quê" (dimensão do conhecimento) de "saber como" (dimensão cognitiva).

Segundo a taxonomia de Bloom revisada (Anderson et al., 2001), cada nível de conhecimento atribuído a um objetivo pedagógico pode corresponder a um nível do processo cognitivo, conforme apresentado na Tabela 1. O aluno "deve ser capaz de lembrar de um fato" e "deve ser capaz de aplicar o conceito".

Tabela 1 - Dimensões dos objetivos educacionais revisados

\begin{tabular}{|l|c|c|c|c|c|c|}
\hline \multirow{2}{*}{\begin{tabular}{c}
\multirow{2}{*}{$\begin{array}{c}\text { DIMENSÃo Do } \\
\text { CONHECIMENTO }\end{array}$} \\
\cline { 2 - 7 }
\end{tabular}} & $\begin{array}{c}\text { (1) } \\
\text { Lembrar }\end{array}$ & $\begin{array}{c}\text { DIMENSÃ } \\
\text { Entender }\end{array}$ & $\begin{array}{c}\text { (3) } \\
\text { Aplicar }\end{array}$ & $\begin{array}{c}(4) \\
\text { Analisar }\end{array}$ & $\begin{array}{c}\text { (5) } \\
\text { Avaliar }\end{array}$ & $\begin{array}{c}(6) \\
\text { Criar }\end{array}$ \\
\hline (A) Factual & $\mathrm{X}$ & & & & & \\
\hline (B) Conceitual & & & $\mathrm{X}$ & & & \\
\hline (C) Procedimental & & & & & & \\
\hline (D) Metacognitivo & & & & & & \\
\hline
\end{tabular}

Nesse sentido, a taxonomia de Bloom revisada foi adotada, neste artigo, para a qualificação das competências demonstradas pelo aluno quando está jogando games.

\section{JOGOS DIGITAIS}

As concepções sobre jogos são várias e abordam desde elementos de entretenimento, passando por elementos pedagógicos até os culturais.

A história da contribuição dos jogos nas outras áreas do conhecimento é longa e continua com maior ênfase com os jogos digitais suportados pelas novas tecnologias, não só com o apoio lúdico, como também com o apoio computacional.

Nesse sentido, jogos digitais podem ser mediadores de aprendizagem e podem contribuir para o despertar de competências, habilidades e melhor compreensão dos conteúdos em diversas áreas. Para Huizinga (2000), "todo jogo é capaz, a qualquer momento, de absorver inteiramente o jogador" em uma espécie de imersão, o que Caillois (1990) corrobora afirmando que "o jogo é essencialmente uma ocupação separada, cuidadosamente isolada do resto da existência".

Segundo Mattar (2010), jogar games "desenvolve a capacidade de deduzir regras pela observação e manipular sistemas complexos, características essenciais para o trabalho em ciências". A atenção e o raciocínio lógico, principalmente em jogos de estratégia, são também elementos estimulados com o uso de jogos digitais. O exercício da atenção nos jogos provoca a imersão do jogador e um estado de extrema concentração na tarefa que está desempenhando.

Outros aspectos importantes acerca dos jogos digitais são a forma de lidar com o erro e a liberdade experimentada pelos jogadores "para descobrir e criar arranjos de aprendizado que funcionem para eles" (Mattar, 2010). No primeiro aspecto, o custo do erro não é tão pesado, porque é possível sempre recomeçar a partir do último ponto que foi salvo (Mattar, 2010), e nesse caso, o erro transforma-se em aliado para uma nova tentativa, de outro modo, sem repeti-lo. 


\section{TRABALHOS CORRELATOS}

A partir de uma revisão sistemática realizada com escopo de busca entre os anos de 2007 e 2012, foram selecionados quatro artigos relevantes que tratam especificamente sobre avaliação formativa e jogos digitais (Galvao; Aranha, 2013), os quais são apresentados a seguir:

Em "Web-based quiz-game-like formative assessment: Development and evaluation" (Wang, 2007), é apresentado um sistema de múltipla escolha baseada em Web quiz-jogo como avaliação formativa chamado de GAM-WATA. A pesquisa envolvendo 165 alunos do ensino fundamental em Taiwan central comparou a eficácia de três diferentes tipos de avaliação formativa em um ambiente de e-Learning: teste de papel e lápis (PPT), teste normal baseado na Web (N-WBT) e GAM-WATA. A partir dos resultados, não há nenhuma diferença significativa entre o grupo PPT e o grupo N-WBT quando comparada a eficácia do estudante nos quatro conceitos de aprendizagem avaliados. No entanto, os estudantes do grupo GAM-WATA tiveram uma eficácia melhor do que aqueles do grupo PPT. Nesse sentido, o autor indica que o uso de jogos no processo de avaliação formativa motiva mais os alunos à participação do que no PPT ou N-WBT.

Matos e Silva (2008), em seu artigo "Desenvolvimento e Avaliação de um Software Educacional Avaliativo: Jogo do Pirata", apresenta um jogo de pergunta e resposta baseado na Web que permite a sua aplicação em diversas disciplinas. O professor é o responsável por povoar o jogo com as questões. "O sistema possui uma monitoração baseada em resultados, como número de acertos e erros e uma monitoração mais detalhada apresentando ao professor".

Thiry et al. (2010), em seu trabalho "Promovendo a Aprendizagem de Engenharia de Requisitos de Software Através de um Jogo Educativo", apresenta uma forma de avaliar alunos de engenharia de requisitos utilizando o jogo educativo ilha dos requisitos. O objetivo do jogo é ajudar o personagem, a fugir da ilha dos requisitos - uma ilha habitada por tribos canibais que tem um vulcão prestes a entrar em erupção. $\mathrm{O}$ jogador deve enfrentar uma série de desafios relacionados ao conteúdo da disciplina de engenharia de requisitos. Os autores concluíram que a maioria dos alunos ficaram motivados e gostaram de jogar o jogo, considerando-o relevante para o aprendizado e que pode ser efetivo para ensinar Engenharia de Requisitos nos níveis de entendimento e compreensão.

O trabalho desenvolvido por Cuomo (2011) "Full Formative Assessment based on Educacional Video Games", trata de uma portal na internet (PlayLearn) para exploração de jogos educativos a longo prazo e testes online. O portal registra os parâmetros de aprendizagem para análise em tempo real pelos professores, com o objetivo de melhorar o processo de aprendizagem. O jogo é baseado na "jornada do herói", no qual o personagem do jogador deve recolher diamantes ao longo da jornada enquanto responde a questões sobre o conteúdo estudado.

Os artigos analisados tratam de avaliação formativa utilizando jogos, mas não definem um método sistemático que qualifica e mede a aquisição de conhecimento dos alunos de modo a subsidiar as ações do professor. Também não exploram competências e habilidades, mas somente o conteúdo estudado.

Nesse sentido, o processo sistêmico, ora proposto, pode contribuir com essa discussão de um modo mais efetivo. Além de propor uma avaliação formativa aplicada em ambiente lúdico e com monitoramento computacional, o processo sistêmico tem elementos que apoiam a instrumentação da avaliação formativa observando conteúdos, competências e habilidades utilizados pelos alunos enquanto jogam. Desse modo, é possível a coleta de dados com respostas dos alunos, gerando informações de desempenho para o professor durante o processo ensino-aprendizagem e não só ao final. 


\section{PROCESSO SISTÊMICO DE INSTRUMENTAÇÃO}

Nesta seção, é apresentado o processo sistêmico proposto por este trabalho, começando pelo método empregado para o seu desenvolvimento e em seguida o detalhamento do processo de instrumentação e a apresentação de um exemplo de aplicação prática, no qual são descritas as etapas de instrumentação em uma disciplina de Administração de Redes em Software Proprietário do curso de Redes de Computadores. $\mathrm{O}$ curso e a disciplina foram escolhidos por conveniência.

\subsection{Método empregado}

A instrumentação da avaliação, aqui proposta, baseou-se nas ideias de Castillo et al. (2011) acerca da avaliação da aprendizagem, que sugerem a exploração de algumas técnicas da observação sistemática, como apoio na tutoria de alunos. Igualmente, foi considerada a proposta de Jorba e Sanmarti (2003) de uma avaliação em três etapas: coleta de dados, análise dos dados e tomada de decisão.

Como método para identificar e qualificar habilidades e competências dos alunos, foi utilizada a taxonomia de Bloom revisada descrita em Anderson et al. (2001) nas suas dimensões do conhecimento e dos processos cognitivos.

Para a associação de alguns gêneros de jogos com as capacitações segundo a taxonomia de Bloom revisada foi utilizado o trabalho de Wangenhein (2012).

\subsection{Instrumentação}

A instrumentação consiste na forma do questionamento ao aluno e do registro das respostas. Para melhor compreensão, classificou-se a forma de questionamento em dois tipos: explícita ou tácita. A primeira estimula respostas diretas do aluno por meio de questões acerca do conteúdo estudado. Explora os vários tipos de instrumentos avaliativos conhecidos, como provas e questionários, em meio ao jogo digital que se adapta às situações de ensino e de aprendizagem propostas pelo professor. A segunda forma, tácita, explora os vários gêneros de jogos digitais como ambiente de exercício e verificação de habilidades do aluno, adquiridas com o estudo dos conceitos estudados nos componentes curriculares.

Os jogos digitais utilizados para a instrumentação de avaliações formativas, são desenvolvidos com os mesmos elementos de jogabilidade de um jogo digital convencional para fins de entretenimento, porém, com o acréscimo de desafios que estimulam o uso das novas habilidades adquiridas.

O jogo digital é o ambiente computacional que apoia a instrumentação da avaliação formativa em suas duas formas de questionamento (explícita e tácita). Fornece não só os recursos computacionais como também o ambiente lúdico para a imersão do aluno. Também registra os dados da interação do jogador com o jogo e o desempenho do aprendiz nas soluções dadas nas formas de questionamentos.

Nesse sentido, segundo seu gênero, os jogos podem ser aplicados para avaliar diferentes níveis de aprendizagem. Os gêneros mais recorrentes para essa finalidade, devido as suas características, são: (a) Adventure; (b) RPG; (c) Estratégia; (d) Simulação; (e) Esportes; (f) Casuais e; (g) Puzzle (Wangenhein, 2012).

$\mathrm{O}$ quadro da Tabela 2, a seguir, demonstra a relação entre as capacitações definidas na dimensão dos processos cognitivos da taxonomia de Bloom revisada e os gêneros de jogos digitais que estimulam a utilização de habilidades que expressam as capacitações. 
Tabela 2 - Relação entre capacitações e gêneros de jogos digitais

\begin{tabular}{|l|c|}
\hline \multicolumn{1}{|c|}{ Dimensão dos processos cognitivos } & $\begin{array}{c}\text { Gênero do } \\
\text { Jogo Digital }\end{array}$ \\
\hline $\begin{array}{l}\text { 6. Criar } \\
\text { Reunir dados para formar algo novo ou reconhecer os componentes de } \\
\text { uma nova estrutura (Gerar, Planejar, Produzir). }\end{array}$ & Simulação \\
\hline $\begin{array}{l}\text { 5. Avaliar } \\
\text { Fazer julgamentos com base em critérios e padrões (Verificar, Criticar). }\end{array}$ & $\begin{array}{c}\text { Estratégia, } \\
\text { Adventure, } \\
\text { RPG, ... }\end{array}$ \\
\hline $\begin{array}{l}\text { 4. Analisar } \\
\text { Dividir um conceito em partes e descrever como elas se relacionam com o } \\
\text { todo (Diferenciar, Organizar, Atribuir). }\end{array}$ & $\begin{array}{c}\text { Estratégia, } \\
\text { RPG, Puzzle, } \\
\text {... }\end{array}$ \\
\hline $\begin{array}{l}\text { 3. Aplicar } \\
\text { Usar um procedimento (Executar, Implementar). }\end{array}$ & $\begin{array}{c}\text { Simulação, } \\
\text { Esportes, ... }\end{array}$ \\
\hline $\begin{array}{l}\text { 2. Entender } \\
\text { Dar um significado ao material ou experiências educacionais (Interpretar, } \\
\text { Exemplificar, Classificar, Resumir, Concluir, Comparar, Explicar) }\end{array}$ & Adventure \\
\hline $\begin{array}{l}\text { 1. Lembrar } \\
\text { Produzir a informação certa a partir da memória (Reconhecer, Relembrar). }\end{array}$ & Casuais \\
\hline
\end{tabular}

Não basta apenas que o jogo pertença a essas categorias, é necessário que algumas premissas sejam observadas para que o jogo digital utilize a forma tácita de questionamento durante um processo de avaliação formativa:

1. A jogabilidade e a solução dos desafios propostos no jogo expressam com clareza o que o professor espera do aluno quanto às suas competências;

2. As atividades avaliativas previstas na forma de questionamento são aqui chamadas de fases, distribuídas conforme o planejamento do professor.

Durante o processo de instrumentação da avaliação formativa no jogo, as habilidades identificadas, segundo o que se espera, são registradas com base em critérios formativos. Os critérios formativos consistem na relação entre as capacitações do domínio do conhecimento e do domínio dos processos cognitivos (Galvao; Aranha, 2013), conforme a taxonomia de Bloom revisada (Anderson et al., 2001).

Tabela 3 - Dimensão do Conhecimento

\begin{tabular}{|l|l|l|}
\hline \multicolumn{2}{|c|}{ Dimensão do Conhecimento } \\
\hline 1) $\begin{array}{l}\text { Conhecimento factual } \\
\text { Informações básicas }\end{array}$ & $\begin{array}{l}\text { Conhecimento de terminologia } \\
\text { Conhecimento de detalhes e elementos } \\
\text { específicos. }\end{array}$ \\
\hline 2) $\begin{array}{l}\text { Conhecimento conceitual } \\
\text { As relações entre as partes de uma } \\
\text { estrutura maior que as fazem } \\
\text { funcionar em conjunto }\end{array}$ & $\bullet \begin{array}{l}\text { Conhecimento de classificações e } \\
\text { categorias }\end{array}$ \\
\hline 3) $\begin{array}{l}\text { Conhecimento de princípios e } \\
\text { generalizações }\end{array}$ \\
\hline Como fazer algo & - $\begin{array}{l}\text { Conhecimento de teorias, modelos e } \\
\text { estruturas }\end{array}$ \\
\hline $\begin{array}{l}\text { Saber raciocinar de modo geral ou } \\
\text { específico }\end{array}$ & $\bullet \begin{array}{l}\text { Conhecimento de técnicas e métodos e dos } \\
\text { critérios para determinar quando usar os } \\
\text { procedimentos apropriados. }\end{array}$ \\
\hline
\end{tabular}


O critério formativo deve, portanto, expressar qualitativamente as competências do aluno de acordo com as capacitações da taxonomia empregada e com as habilidades identificadas e registradas.

\subsection{Exemplo de aplicação prática}

Para exemplificar a aplicação do processo sistêmico de instrumentação da avaliação formativa para o ambiente do jogo digital, considere a disciplina de "Administração de Redes em Software Proprietário", na qual são estudados os conteúdos conforme os módulos: (a) Introdução; (b) Administração do Sistema; (c) Gerenciamento de Serviços e; (d) Núcleo do Sistema.

No seu planejamento, o professor definiu para o módulo "Administração de Sistemas" duas competências a serem adquiridas pelo aluno após a aula sobre o "Agendador de tarefas do Windows Server 2008". O aluno deverá ser capaz de: (Competência 1) Reconhecer as ferramentas de apoio nas atividades assíncronas ou processos interdependentes e; (Competência 2) Implantar e configurar essas ferramentas em laboratório.

Conforme a dimensão dos processos cognitivos da taxonomia de Bloom revisada, os verbos "Reconhecer" da competência 1 e "Implantar" da competência 2, correspondem as capacitações "Lembrar" e "Aplicar" respectivamente.

$\mathrm{Na}$ dimensão do conhecimento, reconhecer requer o "Conhecimento Conceitual" e significa o "conhecimento de classificações e categorias" do que se reconhece. Do mesmo modo implantar e configurar enseja o "conhecimento de técnicas e métodos e dos critérios para determinar quando usar os procedimentos apropriados".

Desse modo, os critérios formativos que atendem às competências definidas pelo professor no planejamento são identificados na Tabela 4, Dimensão do Conhecimento X Dimensão dos Processos Cognitivos, por meio da indicação das relações "B1" que corresponde ao critério 1 (Conceitual-Lembrar) e "C3" que corresponde ao critério 2 (Procedimental-Aplicar).

Tabela 4 - Seleção das capacitações com a definição da relação linha-coluna

\begin{tabular}{|l|c|c|c|c|c|c|}
\hline \multirow{2}{*}{$\begin{array}{c}\text { DIMENSÃo Do } \\
\text { CONHECIMENTO }\end{array}$} & $\begin{array}{c}\text { (1) } \\
\text { Lembrar }\end{array}$ & $\begin{array}{c}\text { (2) } \\
\text { Entender }\end{array}$ & $\begin{array}{c}\text { (3) } \\
\text { Aplicar }\end{array}$ & $\begin{array}{c}\text { (4) } \\
\text { Analisar }\end{array}$ & $\begin{array}{c}\text { (5) } \\
\text { Avaliar }\end{array}$ & $\begin{array}{c}\text { (6) } \\
\text { Criar }\end{array}$ \\
\hline (A) Factual & & & & & & \\
\hline (B) Conceitual & Critério 1 & & & & & \\
\hline (C) Procedimental & & & Critério 2 & & & \\
\hline (D) Metacognitivo & & & & & & \\
\hline
\end{tabular}

O resultado da definição dos critérios formativos é a emissão das expectativas de aprendizagem das dimensões combinadas, ou seja, dos critérios formativos identificados que atendam às competências 1 e 2 do exemplo, onde o Critério 1 atende à Competência 1 e o Critério 2 atende à Competência 2.

Sendo assim, espera-se que o aprendiz seja capaz, respectivamente, de:

(1) estabelecer relações entre as ferramentas de apoio nas atividades assíncronas ou processos interdependentes e entre suas partes que as fazem funcionar em conjunto (conceitual) por meio do domínio sobre a produção da informação certa a partir da memória (lembrar) e;

(2) Implantar e configurar as ferramentas de apoio nas atividades assíncronas ou processos interdependentes em laboratório (aplicar) orientado pelos critérios para determinar quando usar os procedimentos apropriados (procedimental). 
Esses critérios formativos emitidos precisam agora ser quantificados para serem medidos em etapas posteriores. Devido a limitação do espaço do presente artigo, essa discussão será realizada em outro momento oportuno, bem como a descrição dos jogos digitais que estão em desenvolvimento para os testes de consistência do processo.

\section{CONSIDERAÇÕES FINAIS}

A pesquisa ora apresentada compreende, por um lado, a importância dos jogos digitais no contexto educacional, tendo em vista a estreita relação dos alunos com o universo das tecnologias da informação e da comunicação na atualidade. Por outro, evidencia as dificuldades em se avaliar continuamente o conhecimento, considerando o universo complexo, amplo e heterogêneo de alunos, com os quais os educadores têm que lidar cotidianamente.

Nesse sentido, o presente artigo destaca um aspecto da pesquisa cujo intuito é contribuir em duas dimensões da educação. A primeira é o trabalho de avaliação do professor, a fim de que o processo ensino-aprendizagem obtenha resultados mais coerentes com os seus propósitos iniciais. A segunda é o ambiente de avaliação do aluno, a fim de que se torne mais lúdico, e desse modo, menos enfadonho, diminuindo as frequentes queixas sobre avaliações cansativas.

É clara a complexidade do ato de avaliar continuamente, com base nos pesquisadores da área da educação. Sendo assim, o enfrentamento de um aspecto tão importante quanto difícil do processo ensino-aprendizagem, é necessário. Para tanto, a pesquisa envolveu a elaboração de um processo sistêmico de instrumentação da avaliação formativa para o ambiente computacional dos jogos digitais.

A intenção é criar jogos digitais que apoiem a avaliação formativa e, desse modo, auxiliem o professor na regulação da aprendizagem com a consequente melhoria da qualidade e o controle do nível de aquisição de conhecimento dos alunos, uma vez que será possível mensurar, de forma automatizada, o domínio de competências que ele, professor, definiu como importante para os seus aprendizes adquirirem.

São informações que auxiliam o professor a conhecer o desempenho de seus alunos durante o processo ensino-aprendizagem, subsidiando ações individualizadas com o objetivo de sanar deficiências de aprendizagem. Essas ações, ainda durante o processo ensino-aprendizagem, são fundamentais para que o aluno não chegue ao final do período letivo com acúmulos de dificuldades para o atingimento das competências desejadas.

\section{REFERÊNCIAS BIBLIOGRÁFICAS}

ANDERSON et al. A Taxonomy for Learning, Teaching and Assessing: a revision of Bloom's taxonomy of educational objectives. New York: Longman, 2001.

BEHAR, Patrícia Alejandra; SILVA, Ketia Kellen A. Mapeamento de competências: Um foco no aluno da Educação a Distância. RENOTE - Revistas Novas Tecnologias na Educação, v. 10, n. 3, 2012.

CAILLOIS, Roger. Os jogos e os Homens. trad. José G. Palha. Lisboa: Cotovia, 1990.

CASTILlO ARREDONDO, Santiago; GONZÁLES, Luis Polanco; GONZÁLES, Jose Antonio Torres. Formação de tutores: fundamentos teóricos e práticos. Tradução Sandra Martha Dolinsky. Ibpex. Curitiba. 2011.

CUOMO, Salvatore et al. Full Formative Assessment based on Educacional Video

Games. 2011. Disponível em: http://weblab.dmi.unisa.it/. Acesso em: 26/01/2013. 
GALVAO, Glauber; ARANHA, E. Avaliação formativa da aprendizagem com instrumentação em Jogos digitais: Proposta de um framework conceitual. In: VI WORKSHOP DE AVALIAÇÃO E ACOMPANHAMENTO DA APRENDIZAGEM EM AMBIENTES VIRTUAIS (WAVALIA), 6, Campinas, 2013. Congresso Brasileiro de Informática na educação - CBIE. Campinas: UNICAMP, 2013.

HADJI,C. Avaliação desmistificada. Porto Alegre: Artes Médicas, 2001.

HUIZINGA, Johan. Homo Ludens. trad. João P. Monteiro. São Paulo: Perspectiva, 2000.

JORBA, Jaume; SANMARTÍ, Neus. A função pedagógica da avaliação. In: BALLESTER, Margarida (Org.). Avaliação como apoio à aprendizagem. trad. Valério Campos. Porto Alegre: Artmed, 2003. Pág. 23-45.

KRATHWOHL, David R. A Revision of Bloom's Taxonomy: An Overview. In: THEORY INTO PRACTICE, Volume 41, Number 4, pags. 212-264, Autumn 2002. College of Education, The Ohio State University. Disponível em: http://www.celt.iastate.edu/teaching/RevisedBlooms1.html.

MATOS, Marilyn A. E; SILVA, Edy Wilson F. M. Desenvolvimento e Avaliação de um Software Educacional Avaliativo: Jogo do Pirata. RENOTE - Revistas Novas Tecnologias na Educação, v. 6, n. 2, 2008.

MATTAR, João. Games em Educação: Como os nativos digitais aprendem. São Paulo: Editora Pearson Prentice Hall, 2010.

PARCERISA, Artur. Introdução. In: BALLESTER, Margarida (Org.). Avaliação como apoio à aprendizagem. trad. Valério Campos. Porto Alegre: Artmed, 2003. Pág. 11 .

PERRENOUD, Philippe. Avaliação: da excelência à regulação das aprendizagens entre duas lógicas. trad. Patrícia Chittoni Ramos. Porto Alegre: Artmed, 1999.

PIMENTEL, E. P. Um Modelo para Avaliação e Acompanhamento Contínuo do Nível de Aquisição de Conhecimento do Aprendiz. 2006. 218f. Tese de Doutorado Instituto Tecnológico de Aeronáutica, São José dos Campos. Pág. 126-133.

QUINQUER, Dolors. Modelos e enfoques sobre a avaliação: O modelo comunicativo. In: BALLESTER, Margarida (Org.). Avaliação como apoio à aprendizagem. trad. Valério Campos. Porto Alegre: Artmed, 2003. Págs. 15-22.

THIRY, Marcello; ZOUCAS, Alessandra; GONÇALVES. Rafael Queiroz. Promovendo a Aprendizagem de Engenharia de Requisitos de Software Através de um Jogo Educativo. In: SIMPÓSIO BRASILEIRO DE INFORMÁTICA NA EDUCAÇÃO, 21. XXI Simpósio Brasileiro de Informática na Educação - SBIE. João Pessoa: UFPB, 2010.

WANG, Tzu-Hua. Web-based quiz-game-like formative assessment: Developmet and avaluation. 2007. Disponível em: http://www.sciencedirect.com/science/article/. Acesso em: 26/03/2013.

WANGENHEIN, Christiane Gresse von. Ensinando computação com jogos. Florianópolis: Bookess Editora, 2012. 